\title{
Los derechos del lector digital: revisión sistemática de la literatura
}

\section{The rights of digital readers: systematic literature review}

\section{Almudena Mangas Vega}

almumvega@usal.es

\section{Grupo de investigación E-LECTRA: Edición electrónica y Lecto-escritura Digital. Universidad de Salamanca}

\begin{abstract}
Resumen
La irrupción de las nuevas tecnologías ha traído consigo muchos nuevos patrones de comportamiento, entre ellos el cambio de la lectura y los lectores. Junto con los libros electrónicos han aparecido los lectores digitales, y sus derechos. Se presenta una metodología de revisión sistemática de literatura, que está resultando muy útil en distintas áreas del conocimiento en cuanto a reducir tiempo y esfuerzo, con resultados muy acertados. Se analiza, en base a los resultados obtenidos por la revisión sistemática de bibliografía, si el sistema normativo y mercantil ha sabido adaptarse al panorama de los libros electrónicos y si los derechos de los lectores digitales están siendo tenidos en cuenta.
\end{abstract}

\section{Palabras clave}

Lectores digitales, Normativa, Libros electrónicos, Derechos, Revisión sistemática de la literatura, Metodología de búsqueda

\begin{abstract}
The emergence of new technologies has brought with it many new patterns of behavior, including changing readership and reading. Along with e-books have appeared digital readers, and, along with them, their rights. a methodology of systematic literature review, which is proving very useful in different areas of knowledge as to reduce time and effort with very successful results is presented. Based on the results obtained by the systematic literature review, it is analyzed whether the normative and mercantile system has been able to adapt to the panorama of electronic books and if the rights of digital readers are being taken into account.
\end{abstract}




\section{Keywords}

Digital readers, Regulations, E-books, Rights, SLR, Systematic Literature Review, Search Methodology

Recibido: 15/11/2016

Aceptado: 02/12/2016

DOI: http:/ /dx.doi.org/10.5557/IIMEI7-N13-213245

Descripción propuesta: MANGAS-VEGA, Almudena, 2016. Los derechos del lector digital: revisión sistemática de la literatura. Métodos de Información [en línea], 7(13), pp. 213-245.

https://creativecommons.org/licenses/by/4.0/

\section{Introducción}

Más allá de la habitual discusión sobre si el libro en papel sobrevivirá o lo hará el libro electrónico, el hecho es que actualmente ambos conviven.

Estamos en un momento convulso para el entorno del libro. Por un lado, aún se siguen encontrando discusiones enconadas sobre la utilidad de los libros electrónicos o la muerte de los impresos. Y por otro, la edición electrónica de monografías ha tenido en los últimos años un crecimiento notorio, y ha permitido sobrevivir a un gran número de editoriales durante esta última crisis económica.

A este respecto cabe apuntar que se está produciendo un cambio notable en el ecosistema de los libros electrónicos (Cordón-García 2014). Aparecen formatos y plataformas que ofrecen experiencias que van más allá de la simple lectura y que los convierten en elementos completamente nuevos. Eso está produciendo también un cambio en la forma en la que los usuarios se acercan a los libros, en las herramientas que utilizan para leerlos, en el proceso editorial, en los agentes que intervienen en su creación y en los perfiles profesionales necesarios para diseñarlos. Estos cambios también deberían verse plasmados en las normativas y leyes que giran en torno a los libros electrónicos.

Y como agente principal de este nuevo panorama aparece la figura del lector digital, entendiendo éste como el lector que lee libros electrónicos, que conoce el elemento, que, en muchos casos, también lee o leía libros en papel y que 
tiene interiorizados los derechos imprescriptibles del lector que Daniel Pennac (2004) trata en su obra Como una novela y que, más recientemente, gracias a las ilustraciones de Quentin Blake se han hecho casi universales.

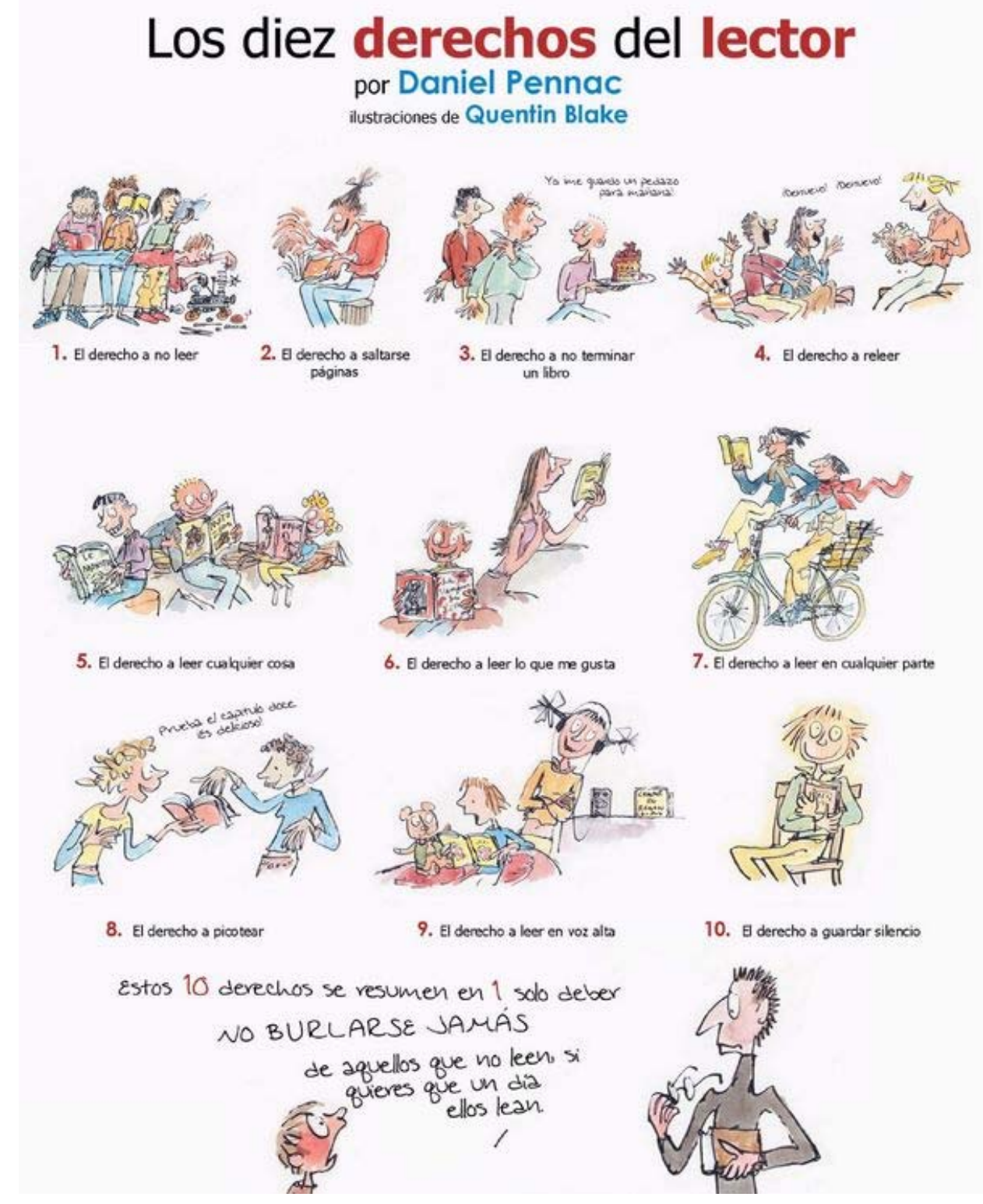

Fig. 1. Los 10 derechos del lector segín Daniel Pennac, ilustrados por Quentin Blake.

Fuente: tULEctura. Universidad de León.

A pesar de que la importancia de un libro recae en su contenido como transmisor de la información, la cultura y el conocimiento y no en el formato o el soporte, la singularidad de los libros electrónicos obliga a tratarlos de forma diferente; también en lo que a normativa - derechos y obligaciones - se refiere. 
Con la aparición de los libros electrónicos, el acceso a todo tipo de monografías ha aumentado considerablemente: en España, en 2007, la edición electrónica aumentó un 88,2\% (Cordón-García y Alonso Arévalo, 2010a); y aunque el aumento no ha vuelto a ser tan notable, no ha cesado en ningún momento. Se está empezando a valorar el potencial de estos libros y en ámbitos académicos, su implantación es toda una realidad. Ante esta realidad, es necesario que la normativa relacionada siga el mismo ritmo para evitar que los lectores digitales se sientan indefensos y con sus derechos disminuidos.

Una primera aproximación al entorno en el que se mueven los lectores digitales muestra su complejidad respecto a la forma en que pueden ser comercializados los libros electrónicos: pago por uso, impresión bajo demanda, compra, etc.; respecto a sus formatos: tangibles como un CD-ROM o intangibles como los ficheros en memoria interna o el acceso y lectura online (Cordón-García et al. 2013); respecto a los derechos y obligaciones adquiridos o las limitaciones a los mismos, como es el caso del DRM (Torres Ripa et al. 2011) o respecto a la definición de su propia naturaleza: en la que habitualmente se mezclan continente y contenido (Mangas-Vega 2014) y de la que dependen restricciones, como el DRM, y tasas, como el IVA.

Otro de los puntos complejos de este entorno es el que tiene que ver con la intimidad de los lectores. Un examen profundo de los derechos de los lectores de Daniel Pennac (Fig. 1) muestra una lectura como una actividad íntima, personal. Algo que en la red puede ser difícil de garantizar.

Se da la circunstancia, además, de que el sentir popular de estos lectores digitales respecto a sus derechos no es del todo bueno (Ávila 2013; Stallman 2010). Las noticias y artículos en los periódicos y los comentarios en las redes sociales muestran malestar y desacuerdo por el tipo de IVA fijado para los libros electrónicos, por las restricciones impuestas tras comprar el producto, por la violación de la privacidad en los datos que se envían desde los dispositivos, ... (Zapata 2011; Streitfeld 2013; Delgado 2012 y García Fernández 2010).

Por todo ello, y con el fin de determinar el estado actual de los derechos de los lectores digitales, en este artículo se realiza una revisión sistemática de literatura, utilizando para ello el método de Revisión Sistemática de la Literatura, en inglés SLR (Systematic Literature Review) adaptada a la singularidad de los elementos buscados. 


\section{Metodología}

La Revisión Sistemática de Literatura (en inglés, SLR) es una metodología surgida en el campo de la Medicina y las Ciencias de la Salud con la intención de ganar experiencia y un conocimiento profundo en un tema de forma rápida pero certera (Kitchenham 2004 y Ramírez 2007). Más recientemente ha resultado ser útil también en otros campos. (Petticrew y Roberts 2008; CruzBenito, Therón y García-Peñalvo, 2016; Radant, Colomo-Palacios y Stantchev 2014 y Toro, Gómez y Rudas 2013). En todos los casos ha demostrado ser un sistema de revisión de literatura científica de gran fuerza.

Siguiendo el modelo de Cruz-Benito (2016) pero adaptándolo a la singularidad de la materia buscada, los pasos a realizar han sido los siguientes:

- Definición de los objetivos y/o preguntas de investigación.

o Se definieron como preguntas de investigación las siguientes:

- RQ1: ¿Cómo están siendo estudiados los derechos de los lectores digitales en el ámbito científico?

- RQ2: ¿Hasta qué punto los derechos de los lectores se ven reflejados en la legislación y la normativa actuales?

- Definición de los criterios de inclusión de los trabajos en la SLR.

o Se definieron como criterios de inclusión, atendiendo a cuestiones técnicas para facilitar el desarrollo de la revisión, los siguientes:

- IC1: Los trabajos hablan expresamente de los derechos, obligaciones o limitaciones de los lectores digitales o de los derechos, obligaciones o limitaciones relacionados con los libros digitales

- IC2: Los trabajos están escritos en español, inglés, francés, italiano o portugués. 
- IC3: Los trabajos han sufrido revisión por pares o están incluidos en Jornadas o Conferencias Técnicas que cuenten con un sistema de revisión científica por pares.

- IC4: Se tiene acceso al texto completo del trabajo.

- Definición de los criterios de exclusión de los trabajos en la SLR.

o De nuevo, atendiendo a cuestiones prácticas, se definieron los siguientes criterios de exclusión:

- EC1: Los trabajos hablan de los libros electrónicos o de los lectores digitales, pero no desde el enfoque de los derechos, obligaciones o limitaciones de los mismos.

- EC2: Los trabajos no están escritos en español, inglés, francés, italiano o portugués.

- EC3: Los trabajos no han sufrido sistema de revisión por pares.

- EC4: No se tiene acceso al texto completo del trabajo.

- Selección de las bases de datos y fuentes de información.

o Como recursos de información se decidió utilizar los siguientes:

- Bases de datos científicas generalistas: WOS (Thomson Reuters), SCOPUS (Elsevier)

- Bases de datos científicas especializadas en el área de Información y Documentación: LISA (ProQuest) y LISTA (EBSCOhost)

- Definición de los términos y las cadenas de búsqueda en los recursos de información:

o A la hora de seleccionar los términos, se han seleccionado en inglés para tratar de abarcar el mayor número de trabajos posibles, ya que las bases de datos revisadas tienen carácter internacional y trabajan con resúmenes, palabras clave y/o títulos en inglés en un porcentaje muy elevado. También se ha eliminado de la cadena el elemento en español "lectores digitales" porque se utiliza con mayor frecuencia para referirse a los dispositivos de lectura electrónicos y emplearlo en la cadena distorsionaría los resultados. Los términos elegidos finalmente han sido: "digital readers", "ebooks", "ebook" and "digital reading" para referirse al entorno de los lectores digitales y 
"rights", "law" y "legacy" como elementos de búsqueda relacionados con la legislación, la normativa y los derechos.

o Debido a las diferencias entre los distintos recursos, y a las posibilidades de búsqueda ofrecidas en los mismos, no ha sido posible utilizar la misma cadena de búsqueda den todos ellos. Así pues, se han definido cadenas con el grado más alto de equivalencia posible a la siguiente:

- [(“digital readers" OR “ebook" OR “ebook" OR “digital reading") AND ("rights" OR "law" OR "legacy")]

- Definición de los límites de búsqueda.

o En función de las posibilidades ofrecidas por cada recurso, se han acotado las búsquedas por:

- Rango temporal: debido a que se trata de un tema en continuo cambio, se ha decidido acotar a trabajos posteriores a 2010 ya que según muchos autores, es en esa fecha cuando la aparición de nuevos dispositivos electrónicos fomenta el auge de los libros electrónicos, los cuales experimentan un rápido aumento en número y lectores. (Cordón-García, Gómez-Díaz y Alonso Arévalo 2011)

- Límite por campos: título, palabras clave y tema.

- Fases de la revisión.

o La revisión ha consistido en un ciclo de dos repeticiones de la siguiente sucesión de fases:

- Fase 1: Ejecución de las cadenas de búsqueda.

- Fase 2: Eliminación de duplicados.

- Fase 3: Revisión de los trabajos restantes atendiendo a los títulos y resúmenes, y aplicando los criterios de inclusión y exclusión definidos previamente.

- Fase 4: Revisión de los trabajos restantes atendiendo al texto completo y aplicando los criterios de inclusión y exclusión definidos previamente.

- Fase 5: Inclusión, si fuera necesario, de trabajos conocidos con anterioridad que no hubiesen sido recuperados en las búsquedas o de aquellos elementos que aparezcan de forma recurrente en trabajos recuperados pero que no 
hayan sido recuperados en la búsqueda. Y repetición del proceso para asegurar la fiabilidad del mismo.

- Evaluación de la calidad de los resultados.

o En el caso de que los elementos recuperados sean demasiado numerosos para poder abordar su revisión, se procederá a ponderar su calidad.

La propia metodología ha sido evaluada según se han ido realizando cada uno de los pasos.

\section{SLR: resultados y discusión}

En base a la aplicación de la metodología establecida, los resultados, según las fases, fueron:

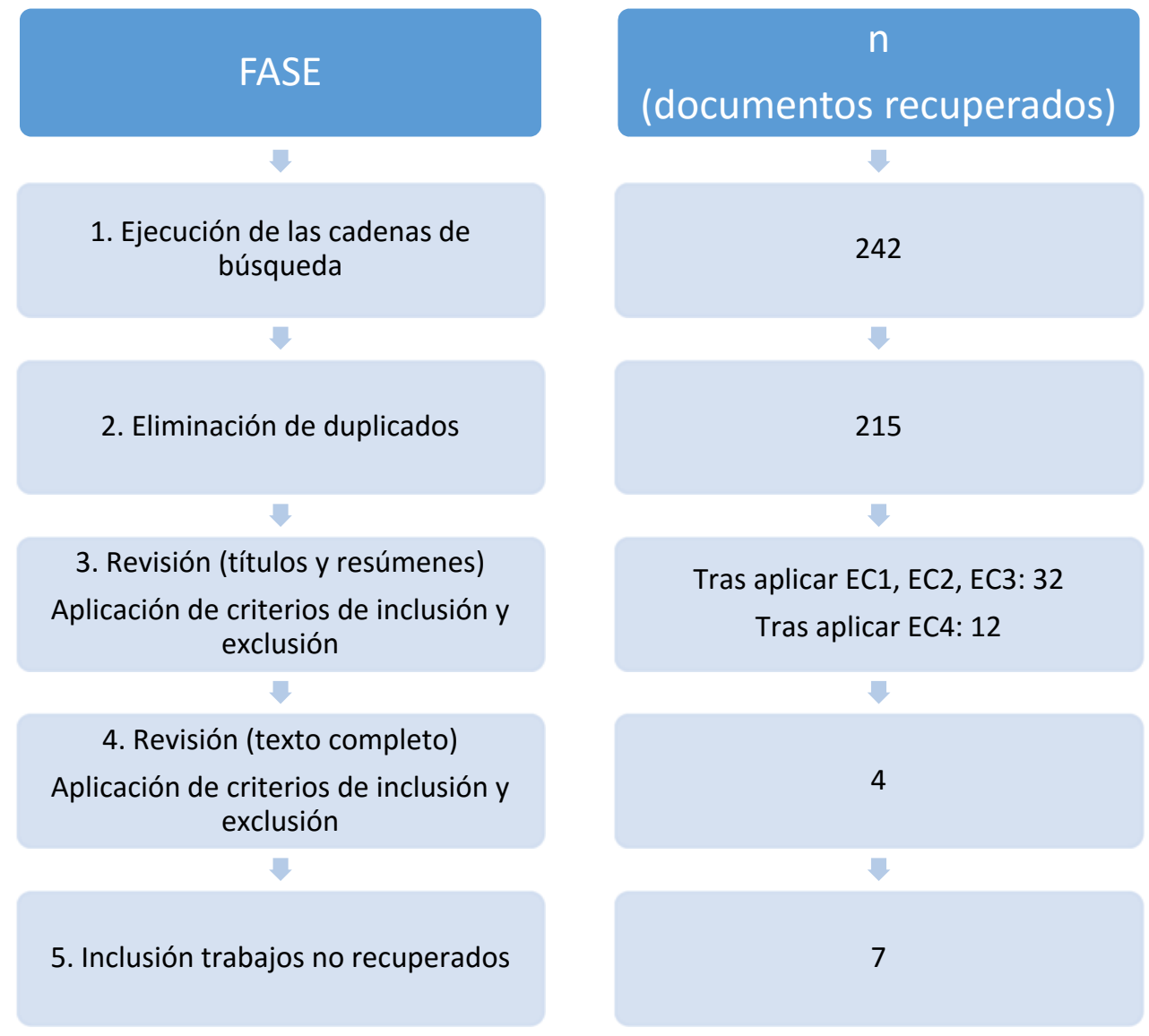

Fig. 2. Fases y resultados en la SLR. 
La metodología de SLR ha permitido reducir los resultados de 242 (número inicial resultante en las búsquedas) a 7 . A su vez, ha permitido mantener la fiabilidad de la revisión ya que cada artículo ha sido analizado, y las eliminaciones han respondido a un bajo nivel de pertinencia.

Respecto al número final de trabajos sobre los derechos de los lectores digitales localizados, hay que indicar que parece un número extremadamente bajo, tratándose además de un tema de actualidad que habitualmente se menciona en artículos de opinión en blogs y periódicos. Y más, si tenemos en cuenta que sólo 4 trabajos han sido localizados en las bases de datos y 3 han sido incluidos manualmente en la fase 5 de la SLR.

Este dato puede tener dos interpretaciones:

- Es un tema que no está siendo tratado con el mismo interés dentro de la comunidad científica que el que se aprecia en la sociedad.

- El tema está siendo estudiado pero las bases de datos científicas no recogen los trabajos.

En cualquiera de los casos, parece necesario profundizar en el tema a un nivel de investigación mayor.

En este caso, para poder tener una aproximación mayor al tema, será necesario revisar más fuentes - quizás no científicas-, que puedan aportar datos que ayuden a visualizar cuál es la situación actual de los derechos de los lectores digitales y si puede ser mejorada. Aun así, la metodología de SLR ha resultado de gran ayuda.

\section{Los lectores digitales y sus derechos}

\subsection{Los libros electrónicos: consideraciones previas.}

El escenario que se estudia está formado por un conjunto de factores relacionados entre sí. Por un lado, hay que hablar de los distintos tipos de soporte de las monografías: papel o digital, y este último, a su vez se puede dividir en otro grupo de formatos: pdf, doc, docx, epub, epub3, código flash en una web, etc. Por otro lado, se tienen que tener en cuenta las implicaciones 
de cada uno de los agentes intervinientes, sin olvidar nunca que el principal de estos (si se puede considerar un agente), para este trabajo, es el usuario final. Uno de los problemas que quizás estén contribuyendo a que los libros digitales no encuentren su sitio en el mercado junto al resto de productos "culturales" es el desconocimiento general de las características y singularidades de los mismos. Este desconocimiento está fomentado, entre otros aspectos, por un problema terminológico que hace que en los países donde se aplica el término "libro electrónico" al continente y al contenido (como es el caso de España o Portugal), o que no distinguen entre libro digital y libro digitalizado, es precisamente donde el desconocimiento existe. Mientras que los países que sí lo distinguen -e-book $<>$ e-reader (países anglófonos), o livres numériques $<>$ livres numerisés (países francófonos)-, llevan la delantera en adecuar sus sistemas a este nuevo paradigma lector (Mangas-Vega 2014).

De hecho, muchos lectores habituales de libros electrónicos han acabado adoptando las denominaciones anglosajonas (ebook para libro electrónico y ereader para lector de libros electrónicos) y evitar así posibles confusiones. Esta apropiación del término anglosajón para salvar limitaciones terminológicas puede apreciarse claramente en las páginas comerciales de numerosos países, como www.casadellibro.com (España), www.scroutz.gr (Grecia), www.halledesprix.fr (Francia).

Por otro lado, es un panorama que no debe ser despreciado por sus cantidades. La edición digital está siendo una vía de escape ante la crisis económica para las pequeñas editoriales que editan en digital y luego imprimen bajo demanda.

\begin{tabular}{|lrrrrrrr|}
\hline & 2009 & 2010 & 2011 & 2012 & 2013 & 2014 & Variación \\
Facturación (millones de €) & 51,2 & 70,5 & 72,6 & 74,2 & 80,3 & 110,0 & $+37,1 \%$ \\
Cuota de mercado (\%) & 1,6 & 2,4 & 2,6 & 3,0 & 3,7 & 5,0 & $+1,3$ puntos \\
\hline
\end{tabular}

Fig. 3. Principales datos de comercialización de libros digitales, 2009-2014. Fuente: El sector del libro en España 2013-2015.

$\mathrm{Y}$, como se muestra en la siguiente figura, la lectura electrónica ha aumentado de forma más notable en formato digital. 


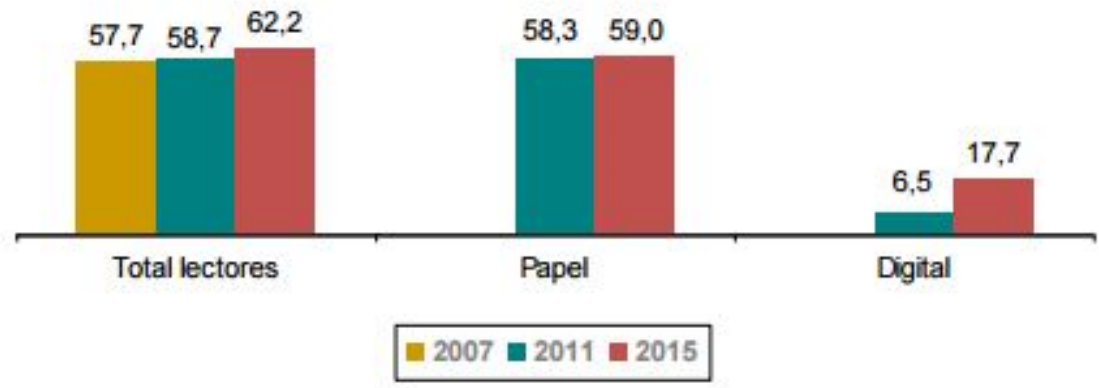

Fig. 4. Porcentaje de lectores en España y su evolución según los formatos que suelen utilizar, 2007-2015. Fuente: El sector del libro en España 2013-2015.

En estos años se han ido sucediendo paulatinamente los elementos utilizados para la lectura: e-readers, tabletas, smartphones, ... pero la gráfica de lectura digital siempre ha sido ascendente.

Como puede observarse, la lectura digital es una realidad en esta Sociedad de la Información y del Conocimiento. Por lo tanto, no deberían existir lagunas respecto a la normativa por la que ha de regirse o a los derechos de sus usuarios.

\subsection{Normativa para el consumidor.}

De una u otra forma, el libro electrónico es un bien de consumo. Y, puesto que lo que le da su identidad y valor son el contenido y la forma -no el formato ni el soporte-, a priori, el sentir popular es que los usuarios deberían tener los mismos derechos que cuando compran un libro impreso (Lionetti 2010).

\section{Normativa pública}

Se considera que el libro impreso es un instrumento fundamental para el desarrollo intelectual y cultural de una persona, tal y como se recoge en la Declaración de los derechos humanos de la Organización de las Naciones Unidas. En base a esto, la Unión Europea permite que se les aplique una tasa reducida (IVA). De esta forma se pretende favorecer su comercio, fomentar el acceso universal a la cultura e infundir en la sociedad la necesidad del desarrollo intelectual al mismo nivel que el físico. 
Esto que está totalmente estandarizado para los libros físicos, no parece ser tan sencillo en el caso de los libros electrónicos. La Unión Europea lleva años trabajando en torno al IVA de los libros electrónicos. Se han generado directivas, modificaciones de las mismas, actuaciones unilaterales por parte de algunos estados miembros, apercibimientos, quejas, denuncias, recursos, ... Parece ser un tema de difícil solución. Tres son los puntos más relevantes hasta el momento en este tortuoso camino:

- La Directiva 2006/112/CE del Consejo de 28 de noviembre de 2006 relativa al sistema común del Impuesto sobre el Valor Añadido indica que entre los bienes y servicios a los que está permitido aplicar el IVA reducido se encuentran "Suministro de libros, comprendido su alquiler por bibliotecas (incluidos folletos, prospectos, impresos afines, álbumes, libros infantiles de pintura, dibujo y coloreado, música impresa o manuscrita, planos y mapas hidrográficos y similares), periódicos y revistas, que no sean íntegra o predominantemente material publicitario", pero en el anexo en el que figuran elementos que se excluyen de ese tipo de IVA en transacciones electrónicas (Anexo II) se indica textualmente: "Suministro de imágenes, texto e información y la puesta a disposición de bases de datos".

Esta contradicción ha generado numerosas interpretaciones por parte de los estados miembros, que, en muchos casos, contaban en su legislación con una definición más actualizada y rigurosa del concepto de libro contemplando también, obviamente, a los libros electrónicos.

Esta situación, que en palabras de López Hurtado y Valentín Ruíz (2016), se ha "planteado como un dislate incomprensible para el sector del libro y para los consumidores" ha devenido en actuaciones unilaterales, como las de Francia y Luxemburgo que decidieron bajar el IVA de los libros electrónicos a su tasa reducida, y en quejas de los editores y usuarios en aquellos países que no hicieron lo mismo.

- En relación con las palabras de estos autores, una de las actuaciones más controvertidas fue la de Francia, que, en noviembre de 2011 decidió de forma unilateral adoptar sistemas de regulación para los libros electrónicos. Su base fue el espíritu de una declaración del Parlamento Europeo del 2006 donde, textualmente se decía que "el copyright y los derechos de protección relacionados debían adaptarse a los nuevos desarrollos económicos como las nuevas formas de explotación"; y su intención era acabar con los 
malentendidos y desacuerdos respecto al trato a las bibliotecas y a los usuarios de libros digitales, con la falta de transparencia en los mercados, con el uso masivo de DRM y con los problemas derivados de la falta de intimidad que pudiera surgir en los entornos digitales (Respingue-Perrin 2013).

- El tercer punto relevante se produce en marzo del 2015, como consecuencia del malestar general a raíz de las actuaciones de Francia y Luxemburgo - o por la no actuación en el mismo sentido en el resto de países-. En esa fecha, el Tribunal de Justicia de la Unión Europea sentenció que no se podía aplicar el IVA reducido a los libros electrónicos porque no hay posibilidad alguna de aplicar un IVA reducido a los "servicios suministrados por vía electrónica" y, lo que ha generado aún más controversia (y desde el punto de vista de los gremios de editores y lectores es un error total), que el "suministro de libros electrónicos no puede considerarse una «entrega de bienes», en el sentido de esta última disposición, porque el libro electrónico no puede ser calificado como bien corporal".

Este hecho es uno de los que más protestas han generado en toda Europa, porque, en el sentir popular, parece que la Comisión Europea no está dando más que pequeños pasos, sin rumbo y a destiempo.

Dejando al margen el punto de vista del IVA, hay que reconocer que en Europa existe una gran lista de organismos oficiales, locales, estatales y a nivel de la Unión Europea donde acudir como consumidores. Son numerosos los documentos localizados. Sin embargo, lo referente al contenido electrónico no está aún muy desarrollado: sí existen varios apartados que regulan la compraventa de productos electrónicos, pero respecto al contenido (los libros propiamente dichos) no hay nada. Y respecto a las nuevas formas de servicios en plataformas de lectura online, tampoco.

Por otra parte, la defensa de los consumidores europeos, que se considera excelente, no tiene sus campos delimitados por completo, algo que muchos lectores digitales agradecen (Rodríguez Rivero 2014).

En un decálogo editado por la Dirección General de Sanidad y Protección de los consumidores de la Comisión Europea en 2005 titulado La protección de los consumidores en la Unión Europea: diezprincipios básicos, se indica: 
- En el apartado 1, que los usuarios europeos pueden comprar lo que quieran y donde quieran (este punto resultaba relevante en el caso de los e-books cuando los impuestos no eran los mismos en todos los países, ya que, por parte de algunos estados miembros habían solicitado que no se pudiesen comprar en otros países, usando las limitaciones por zona, como ya ocurrió con las películas de DVD).

- En el apartado 5 se habla del respeto en los contratos de venta: se estipula que aunque se hubiese firmado un contrato con el distribuidor de libros en el que se aceptara que el importe no se reembolsa en ningún caso, ni siquiera aunque dicho distribuidor no cumpliera su parte del trato, o que no se puede deshacer el contrato sin pagar un plus por ello, etc., la legislación comunitaria blinda frente a este tipo de cláusulas contractuales abusivas, ya que, están taxativamente prohibidas, por lo que en caso de haber firmado, (por inadvertencia o ignorancia), un contrato de estas características, si se ha hecho dentro de un país perteneciente a la UE, la legislación comunitaria le protege frente a esta clase de abusos y podría acudir a sus oficinas para hacer valer sus derechos como ciudadanos comunitarios.

$\mathrm{Y}$ aunque el apartado anterior es una gran protección, en el punto 6 del mismo decálogo, sobre posibilidad de devolución, se recuerda que el usuario dispondrá de un plazo de 7 días laborables para anular el contrato de venta, sin tener que dar ninguna explicación o justificación por ello excepto en el caso de las descargas (como los libros electrónicos), ya que en el momento en el que comienza la descarga se pierde el derecho a retractación o devolución.

Se dio el caso de que muchas empresas utilizaban un doble juego en el que se iniciaba una primera descarga, que no era la del producto elegido propiamente dicho, y luego, si el usuario continuaba con una segunda descarga, había unos costes adicionales. Ante la avalancha de quejas y de sentimiento de indefensión, la Comisión Europea, tal y como muestra en un Comunicado de Prensa del 14 de octubre del 2013, decidió hacer un control sistemático de sitios web en toda la UE para detectar infracciones de la legislación de protección de los consumidores y hacerla cumplir. En dicho control se inspeccionaron los sitios web para:

- Determinar si se encontraba fácilmente y de forma legible la información sobre las características principales de los productos, y si los sitios ofrecían direcciones de correo electrónico de contacto a las que los consumidores pudieran dirigir preguntas o reclamaciones. 
- Verificar si las condiciones generales de los sitios eran correctas.

Los principales problemas que se detectaron fueron los siguientes:

- Cláusulas contractuales abusivas que privan a los consumidores del derecho a emprender acciones judiciales contra la empresa o les deniegan el derecho de indemnización si los productos no funcionan adecuadamente;

- Informaciones poco claras sobre el derecho de retractación: debido a la peculiaridad de las descargas digitales, los comerciantes deben informar a los consumidores antes de la compra de que no podrán cancelar una descarga una vez que esta haya comenzado (el $42 \%$ de los sitios inspeccionados incumplían esta obligación);

- Falta de información obligatoria sobre la identidad del comerciante, especialmente la ausencia de una dirección de correo electrónico, lo que priva a los consumidores de un canal de contacto efectivo con la empresa.

Además del barrido, la Comisión Europea encargó un estudio complementario que puso de manifiesto que no se facilitaba o se ofrecía solo de forma limitada, información sobre restricciones geográficas que puedan aplicarse al sitio. Esta información es esencial para los consumidores que viajan a otros países de la UE, que normalmente esperan tener acceso a sus contenidos digitales y poder utilizarlos sin obstáculos en todo el mercado único. Este estudio también reveló que algunos juegos que se comercializaban como "gratuitos» a menudo requerían el pago de algún importe en una fase posterior, sin que ello se explicara claramente por adelantado. Tales prácticas están dirigidas, con frecuencia, a los niños, las personas más vulnerables entre los grupos de consumidores y pueden darse en los llamados libro-aplicación (García-Rodríguez y Gómez-Díaz 2016).

Como resultado de este estudio y de las medidas legales tomadas a posteriori, de los 332 sitios web analizados el incumplimiento pasó de un 50\% a un 20\% y se sentaron las bases para futuros trabajos similares o a mayor escala.

Sin embargo, este gran trabajo no se ha traducido aún en una modificación de las normas. Tampoco se tiene en cuenta en este caso la singularidad de los libros electrónicos, para los que deberían exigirse requisitos mínimos 
específicos de información, como la compatibilidad con el soporte físico y los programas correspondientes (interoperabilidad), las funciones, la posible aplicación de restricciones geográficas a la utilización del contenido, o la posibilidad de efectuar copias privadas, tal y como se exige en los programas de ordenador o en los juegos electrónicos.

\section{Políticas de las empresas comerciales}

Tras diferentes análisis, se ha podido comprobar que, al menos en Europa, la mayoría de las empresas que comercializan con este tipo de contenidos ofrecen información sobre su política comercial, aunque en muchas ocasiones, esa información no llega al usuario de forma clara. Es el caso de Amazon, cuyos usuarios, en un alto porcentaje, desconocen que lo que han firmado al adquirir sus lectores de libros electrónicos es un contrato de alquiler, no de venta, y que, en la mayoría de los casos ni el lector ni los libros les pertenecen, lo que implicaría que tampoco podrían venderlo o legarlo a sus familiares, como sí harían con su biblioteca física (Bellamy 2014).

\subsection{Limitaciones y extralimitaciones.}

Es, sin duda, el enfoque más estudiado en los trabajos localizados en la revisión de la literatura. Para hacer valer los derechos de los agentes productores (autores, editores, etc.) se ha optado por limitar el uso de los lectores. Y esto, en ocasiones, significa perder "derechos" que al leer en papel tenían.

El punto más problemático y que está generando más desasosiego entre los usuarios es el del Sistema de Gestión de Derechos Digitales -Digital Rights Management, DRM-(Cohen 2009, Schiller 2010 y Sullivan 2016). En la búsqueda de asegurar su contenido muchos editores están limitando el acceso y uso de sus libros digitales mediante este sistema, un conglomerado de programas creado para disminuir las posibilidades de copia (Licher 2009).

Según los detractores, este sistema limita en exceso las funcionalidades del libro y no hace distinción entre copia legal, ilegal o piratería (Serrano Fernández 2015). 
Por otra parte, también empiezan a surgir problemas relacionados con extralimitaciones en el acceso a la intimidad de las personas.

Los libros electrónicos necesitan aplicaciones de lectura para poder ser interpretados. Y muchas de esas aplicaciones recogen y envían a través de internet los datos sobre las lecturas, la velocidad, las notas escritas,... Los usos que se les da después a esos datos son muy diversos, desde recomendaciones de libros a investigaciones científicas (Fundación Germán Sánchez Ruipérez 2014). No todos esos usos son ilícitos, pero, en ocasiones, el usuario no es consultado o no tiene opción de negar esa recogida y reutilización de datos (García Fernández 2010).

A este respecto, la marca española Wolder realizó una encuesta en la que los libros electrónicos y los lectores de libros electrónicos (e-readers) se perfilaban como el gran aliado a la hora de mantener la privacidad sobre lo que uno está leyendo. El 58\% de los encuestados reconocían leer libros digitales precisamente porque así ocultaban lo que estaban leyendo; un 33\% admitió sentir vergüenza al decir lo que leen; un 20\% dijo que si perdiera su e-reader, no lo reclamaría por vergüenza a las obras que lee. Como concluye la marca tras la encuesta, la electrónica de consumo ha de estar al servicio de cada usuario.

\section{Normas y directrices relacionadas}

Respecto a disposiciones legales, la máxima referencia en nuestro país es la modificación de la Ley de Propiedad Intelectual por la Ley 23/2006 que regulariza, aclara y armoniza las disposiciones legales vigentes sobre la materia. En ella se indica que están permitidas las copias de obras literarias, artísticas o científicas sin previa autorización de los titulares de propiedad intelectual, siempre y cuando sea para uso privado del copista, se haga de un material al que el copista ha tenido acceso legítimo y la copia no sea utilizada con fines colectivos ni lucrativos. La copia privada no se aplica a software o programas informáticos.

En el Capítulo II del Título III del Libro I sobre «Los límites a los Derechos de Autor» se establece que los límites de la "copia privada" (conocida 
coloquialmente como "copia de seguridad") a que la copia sea de una obra ya divulgada, realizada por una persona física para su uso privado, que se haya accedido legalmente a la obra, y que la copia no tenga fines ni colectivos ni lucrativos.

En el artículo 31, sobre reproducciones provisionales y copia privada también se indica, textualmente que "no necesita autorización del autor la reproducción, en cualquier soporte, de obras ya divulgadas cuando se lleve a cabo por una persona física para su uso privado a partir de obras a las que haya accedido legalmente y la copia obtenida no sea objeto de una utilización colectiva ni lucrativa, sin perjuicio de la compensación equitativa prevista en el artículo 25, que deberá tener en cuenta si se aplican a tales obras las medidas a las que se refiere el artículo 161. Quedan excluidas de lo dispuesto en este apartado las bases de datos electrónicas y, en aplicación del artículo 99.a), los programas de ordenador."

Atendiendo a este artículo, el DRM estaría privando a los usuarios de poder hacer una copia legal de la obra. La solución a esta contradicción fue la nueva redacción del artículo 31.2, que introduce la novedad de que se obliga a tener en cuenta las medidas tecnológicas de protección de obras (DRM) a la hora del reparto del canon compensatorio por copia privada. Redacción ésta que desde el punto de vista de los colectivos de lectores es totalmente incongruente ya que el uso de medidas tecnológicas impuestas es totalmente incompatible con cobro de un canon compensatorio por la reproducción de obras, aplicable a dispositivos reproductores y grabadores y a todos los soportes y sistemas de almacenamiento, algo que ya está implantado en España desde hace casi una década y que se recoge en el capítulo 25 de la misma Ley. Aunque el Real Decreto Ley 20/2011 de 30 de diciembre, derogó el art.25 "Compensación equitativa por copia privada" de la Ley de Propiedad Intelectual, con lo cual el canon digital queda suprimido. A cambio, en su disposición adicional décima, se introduce una compensación equitativa que el estado abonará a los derechohabientes directamente a cargo de sus presupuestos generales. Es decir, la compensación a los propietarios de los contenidos deja de ser abonada por los fabricantes e importadores de máquinas y soportes de copia para que la asuman todos los ciudadanos, sin distinción, realicen o no copias privadas (López Hurtado y Valentín Ruíz 2016, Jiménez Cano 2013 y Ávila 2013). 


\subsection{Decálogos de los usuarios.}

Los lectores no están de acuerdo con la mayoría de las normativas analizadas ya que entienden que este tipo de legislación es abusiva. En los últimos tiempos han surgido varios movimientos en defensa del lector digital y quizás su mayor aportación ha sido una serie de decálogos o declaraciones de derechos de los lectores de libros digitales.

Entre los defensores más activos se encuentran Dosdoce.com, Electronic Frontier Foundation o Peter Brantley, director de contenidos del Internet Archive.

El trabajo pionero y de los mayores referentes quizás sea el de Javier Celaya y José Antonio Vázquez (2010), cuyos puntos principales se encuentran disponibles para el público en su web Dosdoce.com bajo la forma:

Dodecálogo de derechos del lector de libros digitales:

- 1. Las plataformas de acceso y venta de ebooks no deben comerciar con el historial de compra de los lectores sin su consentimiento previo.

- 2. Aquellas plataformas que quieran reutilizar con fines comerciales el historial de compra de los lectores para mejorar sus sistemas de recomendación de libros o generar ingresos publicitarios relacionados con las compras realizadas deberán comunicar previamente a los lectores qué tipo de información guardan en sus plataformas, por cuánto tiempo y para qué fines comerciales.

- 3. El lector de libros digitales podrá acceder a esta información personal en cualquier momento y borrar su historial en caso de considerarlo oportuno.

- 4. Las plataformas de acceso y venta de ebooks deberán garantizar que los ebooks adquiridos son propiedad de aquellas personas que los han comprado. Tras la polémica decisión de Amazon de entrar en la cuenta de sus usuarios y eliminar los ejemplares digitales vendidos del libro de George Orwell, 1984, por discrepancias con su proveedor, se justifica que exijamos que las plataformas de comercialización de ebooks se comprometan a respetar nuestros derechos como consumidores. Ninguna plataforma o librería virtual debería ser capaz de eliminar de mi cuenta un 
libro ya adquirido o limitar el acceso al mismo sin mi consentimiento expreso.

- 5. En caso de alquiler, pago por lectura o subscripción de cualquier contenido digital, el usuario debería tener una opción a compra perpetua.

- 6. Al igual que en el mundo analógico podemos prestar un libro comprado a un amigo, en el mundo digital deberíamos preservar el derecho a realizar préstamos de libros en cualquier formato y sin coste adicional.

- 7. Se nos debe garantizar la posibilidad de leer cualquier libro de nuestra biblioteca en la nube o plataforma en cualquier dispositivo, sin restricciones ni limitaciones por sistemas, derechos, fronteras, etc., y siempre de una forma amable y legible.

- 8. Las plataformas de acceso y venta de ebooks deberían permitir que las personas que deseen hacer sus compras en un entorno plenamente privado puedan hacerlo sin que sus datos de compra sean almacenados en ningún momento ni comercializados a terceros.

- 9. Los compradores de libros digitales podrán eliminar su historial de compra o alquiler, así como destruir los propios libros adquiridos, en cualquier momento y de forma definitiva sin dejar rastro alguno de su previa existencia en ninguna memoria virtual.

- 10. Los lectores podrán regalar o revender cualquier libro adquirido que ya no se quiera mantener en su biblioteca digital.

- 11. Los lectores podrán subrayar, marcar y hacer anotaciones de forma anónima en sus libros adquiridos. Aquellos lectores que quieran compartir con otros lectores sus anotaciones personales deberán poder hacerlo, pero si en cualquier momento cambian de opinión también podrán retirar las aportaciones prestadas.

- 12. Al igual que podemos mantener nuestro número de teléfono móvil si nos cambiamos de operador, las plataformas deberán garantizar la portabilidad de los datos de los usuarios. Si por cualquier motivo un lector abandona una plataforma deberá poder transportar los libros adquiridos, notas e historial de compra a la nueva plataforma de forma fácil y eficiente.

Y existen más iniciativas, y discusiones sobre estos y otros derechos desde ámbitos divulgativos (Zapata 2011, McSherry y Cohn 2010 Y Millán 2010). 


\subsection{Breve apunte sobre los marcos ilegales.}

Muchas de las malas prácticas de las empresas distribuidoras (García 2013) (que la Unión Europea ya detectó e investigó), la falta de definición y marco establecidos de los libros electrónicos y la ausencia de normativa o legislación en defensa de los derechos de los lectores (también usuarios y consumidores) producen en estos últimos una fuerte sensación de indefensión (Rodríguez Rivero 2014). Este malestar en ocasiones se traduce en comportamientos que rozan o entran de lleno en la ilegalidad. Para una mejor visualización de la pérdida de derechos alegada por los lectores al cambiar de soporte o de formato, se muestran a continuación de forma esquemática los más repetidos en los artículos y comentarios de blogs relacionados:

\begin{tabular}{|l|l|l|l|l|}
\hline DERECHO & $\begin{array}{l}\text { LIBRO } \\
\text { IMPRESO }\end{array}$ & $\begin{array}{l}\text { E-BOOK } \\
\text { con DRM }\end{array}$ & $\begin{array}{l}\text { E-BOOK } \\
\text { sin DRM }\end{array}$ & $\begin{array}{l}\text { E-BOOK de } \\
\text { descarga ilegal* }\end{array}$ \\
\hline Propiedad & Sí & A veces & A veces & Sí \\
\hline $\begin{array}{l}\text { Se puede } \\
\text { prestar }\end{array}$ & Sí & No & No & Sí \\
\hline $\begin{array}{l}\text { Se puede leer en } \\
\text { diferentes } \\
\text { lugares }\end{array}$ & Sí & A veces & Sí & Sí \\
\hline $\begin{array}{l}\text { Se puede re-leer } \\
\text { Sí }\end{array}$ & A veces & Sí & Sí \\
\hline $\begin{array}{l}\text { Privacidad de lo } \\
\text { leído }\end{array}$ & Sí & A veces & A veces & Sí \\
\hline $\begin{array}{l}\text { Privacidad de lo } \\
\text { subrayado }\end{array}$ & Sí & A veces & A veces & Sí \\
\hline
\end{tabular}




\begin{tabular}{|l|l|l|l|l|}
\hline \multicolumn{1}{|l|}{ DERECHO } & $\begin{array}{l}\text { LIBRO } \\
\text { IMPRESO }\end{array}$ & $\begin{array}{l}\text { E-BOOK } \\
\text { con DRM }\end{array}$ & $\begin{array}{l}\text { E-BOOK } \\
\text { sin DRM }\end{array}$ & $\begin{array}{l}\text { E-BOOK de } \\
\text { descarga ilegal* }\end{array}$ \\
\hline $\begin{array}{l}\text { (control sobre...) } \\
\text { anotado }\end{array}$ & Aí veces & A veces & Sí \\
\hline $\begin{array}{l}\text { Se puede } \\
\text { vender y/o legar }\end{array}$ & Sí lo & A veces & A veces & Sí \\
\hline
\end{tabular}

Tabla 1. Derechos libros impresos vs derechos libros electrónicos según los usuarios

* Teniendo en cuenta siempre que en este caso el lector se mueve en la ilegalidad, en esta tabla, sirve a efectos informativos de sus posibilidades y en comparación con las opciones legales.

Si en otros ámbitos de la vida es en el marco ilegal cuando un usuario pierde sus derechos, sería interesante analizar con más profundidad por qué en el caso de los derechos del lector digital, en ese marco es donde se mantienen los mismos derechos de los lectores de libros impresos (o por qué así lo sienten los usuarios).

La ausencia de normativa clara que atienda a los dos puntos de vista (productor/lector) ayuda poco a frenar la piratería. Pero no es el único aliciente que los lectores digitales encuentran en el marco ilegal.

En muchos casos los usuarios deciden - por su cuenta- digitalizar y poner a disposición del resto libros a los que ya no se puede acceder en formato físico. Generalmente son libros que el editor ya no ofrece en sus catálogos (y no quieren volver a ofrecer) o libros cuyos derechos de explotación están en manos de empresas en quiebra. Aunque para todos estos problemas existen pequeños resquicios legales, el acceso a las normas y los tiempos de aplicación hacen que resulte poco práctico. Estos usuarios, en su defensa, alegan actuar (ilegalmente), en pro de la cultura y sin causar daño económico a ninguna empresa. De hecho, autores como Heald (2014) han apuntado a la necesidad de regular el acceso y uso de los libros que se encuentran en esa situación.

En otros casos, muchos usuarios realizan a título personal (ilegal y/o pirata), versiones digitales de libros en papel para que lectores con necesidades 
especiales puedan acceder a un catálogo mayor. No hay que olvidar que los ebooks favorecen de forma extraordinaria el acceso a la información y la cultura en personas con problemas de visión o de alfabetización (Harpur y Suzor 2014), para estos colectivos, las aplicaciones que leen el texto escrito o los audiolibros les ofrecen, según sus propias palabras, "las puertas abiertas a un mundo fascinante que antes no podían ni imaginar".

También se han recogido datos que indican que para muchos usuarios las páginas de préstamo digital en las bibliotecas (préstamo gratuito) son tediosas por la cantidad de pasos que hay que realizar para acceder al libro y poco útiles por el escaso catálogo ofrecido (Frederiksen, et al. 2011). De hecho, muchas de las páginas de descarga ilegal revisadas implican un coste adicional por libro, coste que los usuarios prefieren asumir por su facilidad de uso en vez de acudir al catálogo digital de la biblioteca.

Otros estudios, informes y encuestas (como los elaborados por el Observatorio de Piratería y Hábitos de Consumo) muestran cómo muchos usuarios encuestados aluden a que los ebooks tienen un precio excesivo (algo potenciado por el IVA actual de esos libros) (Delgado 2012, López Hurtado y Valentín Ruiz 2015 y Jimenez, Martin y Palao 2015). De hecho, muchos autores que autopublican sus libros y que han decidido poner un precio menor (entre 1 y 3 euros) han notado un aumento considerable de las ventas (en algunos casos millones de ejemplares) y una reducción aún mayor de la piratería de sus obras.

\section{Conclusiones}

La metodología SLR ha resultado ser muy útil en este caso, dentro del ámbito de la Información y la Documentación. A través de ella se ha podido comprobar que son muy escasos los trabajos que abordan los derechos de los lectores digitales.

Si bien los libros electrónicos son una realidad presente y creciente en la sociedad, y, en consecuencia, también los son los lectores digitales, existen aún lagunas de peso en torno a ellos, como su definición o la legislación relacionada con su uso, su distribución y su precio. 
Las posibilidades que ofrecen los libros electrónicos para las personas con necesidades especiales o las posibilidades de recuperar textos, que en papel ya no se editan ni se localizan por otros cauces, hace que muchos usuarios noveles, por desconocimiento o facilidad, acaben incurriendo en acciones ilegales. Sería interesante estudiar la posibilidad de establecer un marco en el que puedan desarrollarse estos movimientos de buena voluntad.

La gratuidad no es el único elemento que lleva a muchos lectores digitales a utilizar vías ilegales de adquisición de libros electrónicos. De hecho, muchos usuarios pagan por hacerlo. La piratería de ebooks tiene más que ver con los derechos y funcionalidades sobre los libros adquiridos (en su mayoría ligados al DRM) y con la facilidad para acceder a ellos. Estos datos deberían tenerse en cuenta en las acciones anti-piratería de los gobiernos.

Los libros electrónicos ofrecen singularidades que actualmente no son tomadas en cuenta en las normativas, y que afectan a los derechos de sus lectores, derechos en muchos casos, que ya disfrutaban como lectores en papel.

Cada día aparecen nuevas plataformas de contenidos, cada día se crean nuevos y mejores sistemas informáticos que pueden ser alternativas al DRM o que pueden mejorar considerablemente la experiencia de lectura de personas con necesidades especiales, cada día se generan nuevos modelos de creación y edición de libros; los lectores quieren poder disfrutar de todos esos adelantos; los lectores digitales, también. Urge fomentar entre la comunidad científica el estudio - o la visibilidad de los estudios- sobre los derechos de los lectores digitales.

\section{Referencias bibliográficas y fuentes de información}

ÁvILA, A. M., 2013. Contra la industria cultural europea [en linea]. El País. [Consulta: 23/08/2016]. Disponible en: goo.gl/osm4Fm

Barberán Molina, P., 2014. Lo que los editores deben saber sobre la nueva Ley de Propiedad Intelectual. Actualidad Editorial: Observatorio de tendencias y noticias sobre Edición [en línea]. [Consulta: 20/07/2016]. Disponible en: goo.gl/3zG2Yb 
BARKER, P., 1991. Interactive Electronic Books. Interactive Multimedia, 2(1), 11-28.

BELLAMY, C. et al., 2014. Consumer Issues for Planning and Managing Digital Legacies [Leading Edge] [en línea]. IEEE Technology and Society Magazine, 33(3), 2631. [Consulta: 20/07/2016]. ISSN 0278-0097. DOI: 10.1109/MTS.2014.2353751

CASILLI, A. A., 2010. Les liaisons numériques. Vers une nouvelle sociabilité? Paris: Seuil. ISBN 978-2020986373

CELAYA, J. y VÁZQUEZ, J. A., 2010. Derechos de los lectores de libros digitales. Primeras noticias. Revista de literatura, 254, 13-17. ISSN 0034-849X

COHEN, D., 2009. Kindle's DRM Rears its Ugly Head... And It IS Ugly. En: Gear Diary [en línea]. [Consulta: 11/04/2016]. Disponible en: http:/ /geardiary.com/2009/06/19/kindles-drm-rears-its-ugly-head-and-it-is-ugly/

COMISIÓN EUROPEA. Tus derechos en Europa. [Consulta: 25/08/2016]. Disponible en: http://ec.europa.eu/spain/servicios/tus-derechos-eneuropa/index_es.htm

CORDÓN-GARCÍA, J. A., 2014. Manifiesto de la IFLA 2014 por un Internet abierto y transparente. En: José Antonio Cordón-García [en línea]. [Consulta: 16/10/2016].

Disponible

en:

http://diarium.usal.es/jcordon/2014/11/28/1108/

CORDÓN-GARCÍA, J. A. y ALONSO ARÉVALO, J., 2010. El Libro electrónico en el ecosistema de información. Ciencias de la Información, 41(2), 58-68. [Consulta: 23/08/2016].

Disponible

en: http://www.redalyc.org/pdf/1814/181421569008.pdf. ISSN 0864-4659.

CORDÓN-GARCÍA, J.A. y ALONSO ARÉVALO, J., 2010a. Los libros electrónicos: nuevas formas de edición y nuevos modos de lectura. Revista UNE, 20, 21-23.

CORDÓN-GARCÍA, J.A., ALONSO ARÉVALO, J. y MARTÍN RODERO, H., 2010. Los libros electrónicos: la tercera ola de la revolución digital. Anales de Documentación, 13, 53-80. [Consulta: 23/08/2016]. Disponible en: 
http://revistas.um.es/analesdoc/article/viewFile/106991/101681. eISSN 1697-7904

CORDÓN-GARCÍA, J.A., GÓMEZ-DÍAZ, R. y ALONSO ARÉVALO, J., 2011. Gutenberg 2.0: la revolución de los libros electrónicos. Gijón: Trea. ISBN: 978-84-9704-5520

CORDÓN-GARCÍA, J.A., et al., 2013. Libros electrónicos y contenidos digitales en la sociedad del conocimiento: mercado, servicios y derechos. Madrid: Pirámide. ISBN 978-84-3682769-9

CORDÓN-GARCÍA, J.A., et al., 2013a. El Ecosistema del Libro Electrónico Universitario. Madrid: Unión de Editoriales Universitarias Españolas. ISBN 978-849012-285-3

CRUZ-BENITO, J., THERÓN, R., y GARCÍA-PEÑALVO, F. J., 2016. Software architectures supporting human-computer interaction analysis: A literature review. En: Zaphiris, P. y Ioannou, A. (eds.). International Conference on Learning and Collaboration Technologies. Springer International Publishing, pp. 125-136. ISBN 978-3319-39482-4. DOI:10.1007/978-3-319-39483-1_12

DELGADO, C., 2012. ¿Un hotel o una cerveza tienen un IVA reducido? Depende del país donde pague [en línea]. El País. [Consulta: 26/09/2016]. Disponible en: http://economia.elpais.com/economia/2012/06/25/actualidad/1340648587 607760.html

DOCTOROW, C., 2006. How Copyright Broke. Locus Magazine. [Consulta: 26/08/2016]. Disponible en: http://www.locusmag.com/2006/Issues/09DoctorowCommentary.html. ISSN 0047-4959

ESPAÑA. Real Decreto Legislativo 1/1996, de 12 de abril, por el que se aprueba el texto refundido de la Ley de Propiedad Intelectual, regularizando, aclarando y armonizando las disposiciones legales vigentes sobre la materia. BOE, núm. 97, de 22 de abril. [Consulta: 05/08/2016]. Disponible en: https://www.boe.es/buscar/act.php?id=BOE-A1996-8930

ESPAÑA. 1999. Ley Orgánica 15/1999, de 13 de diciembre, de Protección de Datos de Carácter Personal. [Consulta: 02/07/2016]. Disponible en: 
http://www.agpd.es/portalwebAGPD/canaldocumentacion/informes_juridicos/re glamento_lopd/index-ides-idphp.php

ESPAÑA. 2007. Ley 10/2007, de 22 de junio, de la lectura, del libro y de las bibliotecas. BOE, núm. 150, de 23 de junio, pp. 27140-27150. [Consulta: 11/07/2016]. Disponible en: http://www.boe.es/boe/dias/2007/06/23/pdfs/A27140-27150.pdf

ESPAÑA. 2008. Orden PRE/1743/2008, de 18 de junio, por la que se establece la relación de equipos, aparatos y soportes materiales sujetos al pago de la compensación equitativa por copia privada,... BOE, núm. 148, de 19 de junio, pp. 27842- 27844. [Consulta: 13/07/2016]. Disponible en: http://www.boe.es/boe/dias/2008/06/19/pdfs/A27842-27844.pdf

ESPAÑA. Ministerio de Educación, Cultura y Deporte. 2015. Encuesta de hábitos y prácticas culturales España. [Consulta: 22/07/2016]. Disponible en: goo.gl/w70vK4

ESPAÑA. Ministerio de Educación, Cultura y Deporte. 2016. El sector del Libro en España 2013-2015. [Consulta: 22/08/2016]. Disponible en: goo.gl/4ZyxGI

EUROPEAN UNION. European Comission. 2012. Code of EU Online Rights. [Consulta: 10/18/2016]. Disponible en: https://ec.europa.eu/digitalagenda/en/code-eu-online-rights

FEBRE, I., 2013. On Ebook Quality Assurance: Some Questions, Answered. En: Digital Book World [en línea]. [Consulta: 21/08/2016]. Disponible en: goo.gl/bTR8tp

FEDERATION OF EUROPEAN PUBLISHERS. 2015. VAT/GST on Books \& E-books An IPA/FEP Global Special Report. [Consulta: 27/07/2016] Disponible en: http://www.internationalpublishers.org/images/VAT2015.pdf

FISTER, B., 2011. Not sold (Yet) on Ebooks. En: Library Babel Fish: A college librarian's take on technology. Inside Higher Ed. [en línea]. [Consulta: 12/08/2016]. Disponible en: goo.gl/0HGRgj

FREDERIKSEN, L. et al., 2011. Ebooks and interlibrary loan: licensed to fill?. Journal of Interlibrary Loan, Document Delivery \& Electronic Reserve, 21(3), 117-131. ISSN 1540-3572. DOI: 10.1080/1072303X.2011.585102

FRIEDLANDER, J., 2014. A Step by Step Guide to Evaluating Your ePub Files on Kindles, iPads, and Smartphones. En: The Book Designer [en línea]. [Consulta: 
29/08/2016]. Disponible en: http://www.thebookdesigner.com/2014/01/a-stepby-step-guide-to-evaluating-your-epub-files-on-kindles-ipads-and-smartphones /

FUNDACIÓN GERMÁN SÁNCHEZ RUIPÉREZ 2014. Razones de Hellman para defender la ley de privacidad de los lectores de Nueva Yersey. En: Lecturalab [en línea]. [Consulta: 15/08/2016]. Disponible en: goo.gl/ZlPiUn

GARCÍA, C., 2013. Comienza el juicio contra Apple por subir los precios de los ebooks [en línea]. El País. [Consulta: 25/08/2016]. Disponible en: http://cultura.elpais.com/cultura/2013/06/03/actualidad/13702731785403 $\underline{48 . h t m l}$

GARCÍA FERNÁNDEZ, D., 2010. El derecho a la intimidad y el fenómeno de la extimidad. Dereito, 19(2), 269-284. ISSN 2174-0690

GARCÍA-RODRÍGUEZ, A. Y GÓMEZ-DÍAZ, R., 2016. Niños y apps: aprendiendo a leer y escribir en digital. Alabe: revista de investigación sobre lectura y escritura, 13. [www.revistaalabe.com]. ISSN 2171-9624. DOI: 10.15645/Alabe2016.13.6

GÓMEZ-JURADO, J., 2011. La piratería no existe. En: Hipertextual [en línea]. [Consulta: 04/10/2016]. Disponible en: https://hipertextual.com/2011/01/lapirateria-no-existe

HALL, G., 2008. Digitize this Book!: The Politics of New Media, Or Why we Need Open Access Now. Minneapolis: University of Minnesota Press. ISBN 978-0816648719

HARPUR, P. y SUZOR, N. P., 2014. The paradigm shift in realising the right to read: how ebook libraries are enabling in the university sector. Disability and Society, 29(10), 1658-1671. Disponible en: http://eprints.qut.edu.au/78325/

HARRIS, S., 2012. Moving towards an open access future: the role of academic libraries. [Consulta: 21/07/2016]. Disponible en: goo.gl/PP1ab4

HEALD, P. J., 2014. How copyright keeps works disappeared. Journal of Empirical Legal Studies, 11(4), 829-866. ISSN 1740-1461

JIMENEZ, H., MARTIN, B. y PALAO, I., 2015. Observatorio de piratería y hábitos de Consumo de contenidos digitales 2014. S.1.: JfK.[Consulta: 01/09/2016]. Disponible en: goo.gl $/ \mathrm{j} 3 \mathrm{Zm} 2 \mathrm{j}$ 
JIMÉNEZ CANO, R., 2013. Amazon ofrecerá la copia digital de libros comprados en papel [en línea]. El País [Consulta: 21/09/2016]. Disponible en: http:/ / tecnologia.elpais.com/tecnologia/2013/09/04/actualidad/1378310788_261

773.html

JISC., 2009. JISC national e-books observatory project: Key findings and recommendations. Final Report, November 2009. London: JISC. Disponible en: http://observatory.jiscebooks.org/reports/jisc-national-e-books-observatoryproject-key-findings-and-recommendations/

KITCHENHAM, B.A., 2004. Procedures for performing systematic reviews. Keele, UK, Keele University, 33, 1-26. ISSN 1353-7776

KITCHENHAM, B.A. y CHARTERS, S., 2007. Guidelines For Performing Systematic Literature Reviews in Software Engineering, EBSE Technical Report EBSE-2007-01, 2007. Disponible en: http://www.rbsv.eu/courses/rmtw/mtrl/SLR.pdf.

LICHER, V., 2010. The book industry in Germany: looking back at 2009. Publishing Research Quarterly, 26(1), 46-50. ISSN 1053-8801

LIONETTI, J., 2010. Declaración de los derechos del lector. En: Libros en la nube [en línea]. [Consulta: 25/07/2016]. Disponible en: http://librosenlanube.blogspot.com.es/2010/09/declaracion-de-los-derechos-dellector.html

LÓPEZ HURTADO, M. y VALENTÍN RUÍZ, F.J., 2016. Diez años de controversia en torno al IVA del libro electrónico. ROED: Revista online de estudiantes de Derecho, 5. [Consulta: 23/08/2016]. Disponible en: https://www.uam.es/otros/roed/docs/cultura5.2016.pdf

LYNCH, C., 2001. The battle to define the future of the book in the digital world. First monday, 6(6). [Consulta: 16/07/2016]. Disponible en: http://firstmonday.org/ojs/index.php/fm/article/view/864/773 ISSN 13960466

MANGAS-VEGA, A., 2014. Un pdf no es un libro electrónico. Vegajournal.org, 10(1), 40-53. [Consulta: 22/10/2016]. Disponible en: 
http:/ /www.vegajournal.org/content/archivio/66-2014-04-anno-x-numero-1/323un-pdf-no-es-un-libro-electronico. ISSN 1826-0128

MANRIQUE, W. y SILVA, R., 2014. Los eslabones de la creación y la producción de un libro [en línea]. El País. [Consulta: 22/10/2016]. Disponible en: http://elpais.com/elpais/2014/06/11/media/1402515879_970524.html

MARKMONITOR. 2016. Pirated digital content. [Consulta: 22/08/2016]. Disponible en:https://www.markmonitor.com/download/Infographics/MarkMonitor.infograp hic.IntellectualPiracy.pdf

McGill, M. L., 2003. American Literature and the Culture of Reprinting, 1834-1853. Philadelphia: University of Pennsylvania Press.

MCSHERRY, C. y COHN, C., 2010. Digital Books and Your Rights: A Checklist for Readers. En: Electronic Frontier Foundation. [Consulta: 23/07/2016]. Disponible en: https://www.eff.org/wp/digital-books-and-your-rights

MILLÁN, J. A., 2010. Los derechos del lector (digital). En Libros y bitios [en línea]. [Consulta: 15/10/2016]. Disponible en: http://jamillan.com/librosybitios/2010/09/los-derechos-del-lector-digital/

MORA, M. y DONCEL, L., 2014. Bruselas castiga a Luxemburgo y Francia por el IVA del libro digital [en línea]. El País. [Consulta: 22/10/2016]. Disponible en: http://cultura.elpais.com/cultura/2013/02/21/actualidad/13614816220112 $\underline{92 . h t m l}$

PENNAC, D. 2004. Como una novela. S.1.: Grupo Editorial Norma. ISBN: 9789580494928

PETTICREW, M., y ROBERTS, H., 2008. Systematic reviews in the social sciences: $A$ practical guide. Hoboken, New Jersey, John Wiley \& Sons. ISBN 978-1405121101

PIESING, M., 2013. Is the Book Discoverability Bubble Ready to Pop? [en línea]. En: Publishing Perspectives. [Consulta: 20/08/2016]. Disponible en: http:/ / publishingperspectives.com/2013/02/is-the-book-discoverability-bubbleready-to-pop/ 
RADANT, O., COLOMO-PALACIOS, R., y STANTCHEV, V., 2014. Analysis of Reasons, Implications and Consequences of Demographic Change for IT Departments in Times of Scarcity of Talent: A Systematic Review. International Journal of Knowledge Management (IJKM), 10(4), 1-15. ISSN 548-0666 EISSN: 15480658

RAMÍREZ, R. 2007. Calidad de vida relacionada con la salud como medida de resultados en salud: revisión sistemática de la literatura. Revista Colombiana de Cardiología, 14(4), 207-222. ISSN 0120-5633

RESPINGUE-PERRIN, S., 2013. Too early, too fast? The regulation of the eBook market in France and its possible effects on EU libraries. Liber Quarterly, 23(2). ISSN 1435-5205

RODRÍGUEZ RIVERO, M. 2014. La larga mano del cibertendero global [en línea]. En: El País. [Consulta: 13/09/2016]. Disponible en: http://cultura.elpais.com/cultura/2014/06/10/babelia/1402411894_062954.html

ROMERO-OTERO, I.S., GIMÉNEZ TOLEDO, E. y MARTÍN-GONZÁLEZ, J.C., 2010. El e-book científico-técnico en el mercado español a partir del análisis de las agencias de suscripciones. El profesional de la Información, 19(1), 21-27. ISSN 16992407

SCHILLER, K., 2010. A Happy Medium: Ebooks, Licensing, and DRM. Information Today. 27(2). 1, 42, 44. ISSN 8755-6286

SERRANO FERNÁNDEZ, M., 2015. El actual marco legal de los límites a los derechos de autor en favor de las bibliotecas: razones de su inadecuación al entorno digital y propuesta de reforma legislativa. Boletin mexicano de derecho comparado, 48(143), 799-829. ISSN 2448-4873

SHELBURNE, W. A., 2009. E-book Usage in an Academic Library: User Attitudes and Behaviors. Library Collections, Acquisitions, \& Technical Services. 33. 59-72. ISSN 1464-9055

STALLMAN, R., 1997. The Right to Read. En: El sistema operativo GNU [en línea]. [Consulta: 15/08/2016]. Disponible en: https://www.gnu.org/philosophy/right-toread.en.html

STREITFELD, D., 2013. El libro sabe si el niño ha estudiado [en línea]. El País [Consulta: 25/06/2016]. Disponible en: 
http:/ / tecnologia.elpais.com/tecnologia/2013/05/01/actualidad/1367408335_779 695.html

STRIPHAS, T.G., 2009. The Late Age of Print: Everyday Book Culture from Consumerism to Control. New York: Columbia University Press. ISBN 978-0231148146

SULLIVAN, J., 2016. Letter to the Boston Public Library [en línea]. En: Free Software Foundation. [Consulta: 02/09/2016]. Disponible en: http://www.fsf.org/campaigns/drm.html/bpl.html

TORO, A. O., GÓMEZ, L. M. y RUDAS, J. S., 2013. Revisión sistemática de literatura. Caso de estudio: Modelamiento de un par deslizante con fines de predecir desgaste. Prospectiva, 11(1), 50-58. ISSN 1692-8261. EISSN 2216-1368

TORRES RIPA, J. et al., 2011. El copyright en cuestión: diálogos sobre propiedad intelectual. Bilbao: Deusto Digital Publicaciones. ISBN 978-84-9830-301-8

UNIÓN EUROPEA. Directiva 1999/44/CE del Parlamento Europeo y del Consejo. [Consulta: 25/07/2016]. Disponible en: goo.gl/PgY03I

UNIÓN EUROPEA. Directiva 2006/112/CE del Consejo de 28 de noviembre de 2006 relativa al sistema común del impuesto sobre el valor añadido. [Consulta: 27/08/2016]. Disponible en: goo.gl/RWFDGT

UNIÓN EUROPEA. Directiva 2011/83/UE, del Parlamento Europeo y del Consejo de 25 de octubre de 2011 sobre los derechos de los consumidores. [Consulta: 25/07/2016]. Disponible en: goo.gl/VGLrxG

UNIÓN EUROPEA. Directiva 85/577/CEE del Consejo. [Consulta: 25/07/2016]. Disponible en: goo.gl/mm1xRj

UNIÓN EUROPEA. Directiva 93/13/CEE del Consejo. [Consulta: 25/07/2016]. Disponible en: goo.gl/hqGGhf

UNIÓN EUROPEA. Directiva 97/7/CE del Parlamento Europeo y del Consejo. [Consulta: 25/07/2016]. Disponible en: goo.gl/0Dc39k

UNIÓN EUROPEA., 2013. Una mejor protección para los consumidores europeos a la hora de descargar juegos, música, vídeos y libros electrónicos. [Consulta: 27/07/2016]. Disponible en: http://europa.eu/rapid/press-release_IP-13-937_es.htm 
ZAPATA, J.L., 2011. Los Derechos de los Lectores de Libros Digitales. En: Hipertextual [en línea]. [Consulta: 11/06/2016]. Disponible en: https://hipertextual.com/2011/03/los-derechos-de-los-lectores-de-libros-digitales 